COLO-HEP-410

\title{
Optimizing the Chiral Properties of Lattice Fermion Actions
}

\author{
Thomas DeGrand, Anna Hasenfratz, and Tamás G. Kovács \\ Physics Department, University of Colorado, \\ Boulder, CO 80309 USA
}

(the MILC collaboration)

\begin{abstract}
We describe a way to optimize the chiral behavior of Wilson-type lattice fermion actions by studying the low energy real eigenmodes of the Dirac operator. We find a candidate action, the clover action with fat links with a tuned clover term. We present a calculation of spectroscopy and matrix elements at Wilson gauge coupling $\beta=5.7$. The action shows good scaling behavior.
\end{abstract}




\section{Introduction}

There is an increasing accumulation of evidence from lattice simulations of the importance of the topological properties of the QCD vacuum [1]. The (lattice) pure gauge QCD vacuum is filled with instantons of average radius $\sim 0.3 \mathrm{fm}$, with a density of about $1 \mathrm{fm}^{-4}$, and they are responsible for a large part of chiral symmetry breaking in QCD [2, 3]. The extent to which the complete continuum phenomenology of instanton effects in hadronic physics is actually realized by $\mathrm{QCD}$, and can be studied using lattice simulations, remains an open question, but its outline is present.

Lattice artifacts associated with instantons also seem to be connected to some of the difficulties of numerical simulations with quenched QCD, namely, eigenmodes of the Dirac operator which occur away from zero bare quark mass, and which spoil the calculation of the fermion propagator, the so-called "exceptional configurations" [4, 5].

In this work we wish to look at topology and fermions on the lattice from a different perspective: given that instantons are responsible for chiral symmetry breaking, is it possible to optimize the lattice discretization of a fermion action with respect to its topological properties? Our goal is to shrink the range of bare quark mass over which the low lying real eigenmodes of the Dirac operator occur, on background gauge field configurations which are typical equilibrium configurations at some gauge coupling.

The action we propose is the standard clover action, except that the gauge connections are replaced by APE-blocked [6] links, and the clover coefficient is tuned to optimize chiral properties. Specifically,

$$
\begin{aligned}
S & =\sum_{n}(m+4) \bar{\psi}(n) \psi(n) \\
& -\frac{1}{2} \sum_{n \mu}\left(\bar{\psi}(n)\left(1-\gamma_{\mu}\right) V_{\mu}(n) \psi(n+\mu)+\bar{\psi}(n)\left(1+\gamma_{\mu}\right) V_{\mu}^{\dagger}(n-\mu) \psi(n-\mu)\right. \\
& +\frac{C}{2} \sum_{n, \mu, \nu} \bar{\psi}(n) i \sigma_{\mu \nu} P_{\mu \nu}(n) \psi(n)
\end{aligned}
$$

with

$$
\begin{array}{rc}
V_{\mu}^{(n)}(x)= & (1-c) V_{\mu}^{(n-1)}(x) \\
& +c / 6 \sum_{\nu \neq \mu}\left(V_{\nu}^{(n-1)}(x) V_{\mu}^{(n-1)}(x+\hat{\nu}) V_{\nu}^{(n-1)}(x+\hat{\mu})^{\dagger}\right. \\
& \left.+V_{\nu}^{(n-1)}(x-\hat{\nu})^{\dagger} V_{\mu}^{(n-1)}(x-\hat{\nu}) V_{\nu}^{(n-1)}(x-\hat{\nu}+\hat{\mu})\right)
\end{array}
$$


with $V_{\mu}^{(n)}(x)$ projected back onto $S U(3)$ and $V_{\mu}^{(0)}(n)=U_{\mu}(n)$ the original link variable. We take $c=0.45$ and $N=10$ smearing steps, chosen because of our previous work in instantons [3]. This choice of parameters is not unique and might not even be optimal. $P_{\mu \nu}$ is the usual clover set of links, but built of the $V_{\mu}$ 's. At Wilson gauge coupling $5.7-5.8$, the best choice is $C=1.2$, and it decreases to the tree-level $C=1$ value at larger $\beta$. We will also quote simulation data in terms of the hopping parameter, $\frac{1}{2 \kappa}=m+4$.

The optimized action has a spread of low lying real eigenmodes with respect to the bare quark mass which is less then a third of the usual Wilson action. In terms of the square if the pion mass the spread is about three times smaller for the optimized action than for the standard Wilson action. The action has other good features, as well: the renormalization factors connecting lattice quantities to their continuum values appear to be very close to unity. The action also appears to require only about half the number of sparse matrix inversion steps as the usual clover action (at equivalent values of physical parameters).

It is possible to tune the standard clover action (with the original links used as connections) to improve the interaction of quarks with instantons. This choice is not the best one. It happens that the best value of $C$ for $\beta<6.0$ is large enough that a new class of exceptional configurations appear. These configurations compromise simulations. The connection between exceptional configurations and instantons is explored in detail in a companion work by us [5].

The action we propose is completely unimproved in its kinetic properties, so its dispersion relation and heavy quark mass artifacts are identical to those of the Wilson action. It would be very easy to improve it by beginning with a more complicated free fermion discretization.

Fermion actions with fat links have received considerable attention in the past year. The first use of them we know of (although with a different motivation) was in the simulations of QCD on cooled gauge fields by the MIT group [7]. The MILC collaboration [8], Orginos and Toussaint [9] and Lagaë and D. K. Sinclair 10 have shown that modest fattening considerably improves flavor symmetry restoration in simulations with staggered fermions. We have used calculations of staggered spectroscopy on highly smoothed gauge configurations to compare chiral symmetry breaking in $S U(2)$ gauge theory and in instanton backgrounds [1]. Finally, all fixed point actions and approximate fixed point actions we know of 12, 13] for fermions seem to incorporate fat links. Fixed point fermions realize the index theorem and retain chiral symmetry at nonzero lattice spacing [14], and so one way of viewing a fat link action is as an approximate FP action, which includes its chiral properties but not its 
kinetic ones.

An apparent drawback of fat link actions is the lack of a transfer matrix between consecutive time slices. N APE steps can mix links up to $\pm N$ timeslices away and a strict transfer matrix cannot be defined on time slices closer than $2 N$ lattice spacings (20 in our case). In practice we are concerned only with the exponential decay of correlation functions and we found asymptotic decay after 3-5 time slices in our simulation. This is expected if we realize that APE smearing is basically a random walk whose range can be estimated as $\sqrt{N} c \approx$ 1.4 .

The outline of the paper is as follows: In Section 2 we review the continuum index theorem and describe how lattice fermions fail to reproduce it. We then describe how we find the real eigenmodes of the Wilson-Dirac operator. All this is basically a review, and experts may skip it. In Section 3 we describe the tests we performed to tune the action. Section 4 describes a calculation of spectroscopy and matrix elements at $a T_{c}=1 / 4$ (Wilson gauge coupling $\beta=5.7$ ) using the new action.

\section{Measuring the Chiral Properties of Wilson- like Fermions}

The reader might recall that the local topological density

$$
q(x)=\frac{1}{32 \pi^{2}} \epsilon_{\mu \nu \rho \sigma} F_{\mu \nu} F_{\rho \sigma}
$$

is related to the divergence of the flavor singlet (with $n_{f}$ flavors) axial-vector current

$$
\partial_{\mu} \bar{\psi} i \gamma_{\mu} \gamma_{5} \psi=2 m \bar{\psi} i \gamma_{5} \psi+2 i n_{f} q .
$$

Integrating over all $x$, this relation implies a connection between the topological charge $Q=\int d^{4} x q(x)$ and a mode sum,

$$
Q=m \operatorname{Tr} \bar{\psi} \gamma_{5} \psi=m \operatorname{Tr} \gamma_{5} \frac{1}{\gamma \cdot D+m}=m \sum_{s} \frac{f_{s}^{\dagger} \gamma_{5} f_{s}}{i \lambda_{s}+m}
$$

where $f_{s}$ are the eigenfunctions of the (antihermetian) Dirac operator $\gamma \cdot D$, $\gamma \cdot D f_{s}=i \lambda_{s} f_{s}$, with $\lambda_{s}$ real. The property $\left\{\gamma_{5}, \gamma \cdot D\right\}=0$ leads to the condition that $f_{s}^{\dagger} \gamma_{5} f_{s}=0$ if $\lambda_{s} \neq 0$, and if $\lambda_{s}=0$ we may choose $f_{s}$ to have a definite chirality, $\gamma_{5} f_{s}= \pm f_{s}$. Thus it follows that

$$
Q=\sum_{s, \lambda_{s}=0} f_{s}^{\dagger} \gamma_{5} f_{s}=n_{+}-n_{-}
$$


where $n_{+}$and $n_{-}$are the number of zero eigenmodes with positive and negative chirality. This is the index theorem.

On the lattice, essentially every statement in the preceding paragraph is contaminated by lattice artifacts 15. Here we focus on Wilson-like fermions, where chiral symmetry is broken by the addition of terms proportional to the Dirac scalar and/or tensor operators. The Wilson or clover fermion action analog of $\gamma \cdot D, D_{w}$, is neither Hermetian nor antihermetian and its eigenvalues are generally complex. $D_{w}$ can also have real eigenvalues. These real eigenvalues usually do not occur at zero bare quark mass. Their locations spread across a range of quark mass values.

On smooth, isolated instanton background configurations, the location of the real eigenmode varies with the size $\rho$ of the instanton. For large instantons, the low lying real mode occurs close to zero quark mass. Accompanying these nearzero modes are a set of modes which do not cluster around $m_{0}=0$, but around $-a m_{0} \simeq O(1)$. These are "doubler modes." As one decreases the instanton size, the eigenmodes at $a m_{0} \simeq 0$ shift towards negative quark masses, approaching the doubler modes. This $\rho$-dependent mass shift and the location of the doubler modes all depend on the particular choice of lattice fermion action 15, 16, 17]. The shift of the low lying real eigenmode for a given instanton size for the Wilson action is larger than for the clover action, which has better chiral properties. Nevertheless, for both actions, as the instanton size decreases, sooner or later the low energy eigenmode shifts to large negative quark mass, approaches the doublers and eventually annihilates with one of the doublers - the fermion does not see the instanton any longer.

On equilibrium background configurations, the shift of the eigenmode with $\rho$ is accompanied by an overall $\beta$-dependent mass shift. On top of that there is the spread of the eigenmodes according to the sizes of the background instantons. The spread of these low energy modes, if distinguishable from the doublers, characterize the amount of explicit chiral symmetry breaking of the fermionic action. On configurations with small lattice spacing the typical instanton is large in lattice units. The spread of the low energy modes is small and they are well separated from the doublers. The explicit chiral symmetry breaking of the fermionic action is small and controlled. On configurations with large lattice spacing the instantons are small (if present at all). The spread of the low lying modes is large, close to or overlapping with the doubler modes. The hadron spectrum on these configurations could numerically be similar to the continuum spectrum, but the physical mechanism behind it is very different from continuum QCD.

Determination of the locations of the real roots of $D_{w}+m$ in a particular 
background gauge configuration can be done very simply. We approximate

$$
P\left(m_{0}\right)=\left\langle\bar{\psi} \gamma_{5} \psi\right\rangle=\operatorname{Tr}\left(D_{w}+m_{0}\right)^{-1} \gamma_{5}
$$

with a noisy estimator: cast a random vector $\eta_{i}$ on each site $i$, compute $\left(D_{w}+\right.$ $\left.m_{0}\right) \chi=\eta$ and measure

$$
P\left(m_{0}\right)_{\eta}=\sum_{i j} \operatorname{Tr} \bar{\eta}_{i} \gamma_{5} \chi_{i}
$$

as a function of $m_{0}$. The eigenmode of $D_{w}$ is located at $-m_{0}$ and its appearance is signaled by the appearance of a pole in $P\left(m_{0}\right)$. This is a variant on the standard method of measuring $\langle\bar{\psi} \psi\rangle$ in a lattice simulation. It has also seen considerable use by the Fermilab group [4] in their studies of exceptional configurations (they use a flat source $\eta_{i}=$ constant, not a noisy source). The eigenfunction itself of a particular mode can be found by performing the inversion for a test mass very close to an eigenvalue. Then

$$
\chi=\sum_{s} \frac{f_{s}\left(f_{s}^{\dagger}, \eta\right)}{\lambda_{s}+m_{0}}
$$

is saturated by the mode closest to the pole, and $\chi^{\dagger} \chi$ is (proportional to) the probability density for that mode.

\section{Testing and Tuning Actions}

We study the locations of low energy real eigenmodes in equilibrium (quenched) gauge configurations. Here there are two issues: First, do the low lying modes separate from the doubler modes, and second, what is the spread of the low lying modes.

The first question is important because, if the low lying modes do not separate from the doubler modes, then the physics of chiral symmetry breaking with a lattice cutoff is different than in the continuum. The lattice theory is no longer just a crude approximation to the continuum, and one cannot speak of chiral symmetry breaking as being induced by instantons. Chiral symmetry is certainly broken in QCD for any value of the cutoff, including the strong coupling limit [18], but the mechanism does not involve instantons.

Note that if the low lying modes do not separate from the doubler modes, it does not make sense to talk about the spread of the low lying modes.

In this paper we restrict ourselves to clover-like actions. Our goal is to tune the clover coefficient for good chiral behavior, i.e. to minimize the spread of the 
low lying modes. We could attempt this program on the original configurations. However, it is known that rough gauge configurations with large clover coefficient are plagued by exceptional configurations. This problem is greatly reduced if the links of the configurations are smoothed by a series of APE smearing steps [5]. We also know from previous work [11] that an action with fat links is insensitive to short distance fluctuations, but still knows about instantons and the additional long distance behavior of the gauge field responsible for confinement. So we will begin with a fat link action of Eqn. 11. Throughout this paper we create the fat link by 10 APE smearing steps with smearing coefficient $c=0.45$.

Figure 11 shows the spread of the real eigenmodes at $\beta=5.8$ as the function of the bare quark mass for clover coefficients $C=0.0$ (Wilson action), $C=1.0$, $C=1.2$ and $C=1.4$. The eigenmodes were located using the pseudoscalar density function of Eqn. 8 on $408^{4}$ configurations. The horizontal ranges of the histograms are equal to the range the eigenmode search had been performed. To the right of the distributions is the confining phase. If one measures the pion mass in the positive mass region and extrapolates $m_{\pi}^{2}$ to zero with the bare mass $m_{0}$, one finds that the bare mass at which $m_{\pi}=0$ lies at a value $m_{c}$ located within the range of the low-lying real eigenmodes [5]. If a particular configuration has an eigenmode with $m_{0}>m_{c}$, and if one attempts to compute the quark propagator at $m=m_{0}$, the propagator will be singular. This is (one kind of) exceptional configuration, and at a minimum it compromises the statistical averaging process inherent in the Monte Carlo simulation process. To the left of the distributions, in the negative quark mass region, are the doublers. In Figure 11 there is no indication of them, since the doublers did not show up within the range we scanned for eigenmodes. It appears that the doublers and low lying modes are well separated at $\beta=5.8$. This seems to be in contradiction with the statement of Ref. [19], where the authors claim that the real modes cover the whole investigated negative quark mass range. Of course, as there is a non-zero probability of finding an eigenmode anywhere, our claim is only that the distribution is strongly peaked at the low lying modes and the doublers are well separated. Since in Ref. 19 no distribution histogram is published, we cannot tell if there is a real discrepancy between the results or it is the question of interpreting the data. One should also note that in 19 thin link actions were used, so we are really discussing two different actions. We will return to this question in [5].

To quantify the spread of the low energy real modes, in figure 2 a we plot the average real eigenmode location as the function of the clover coefficient. The error bars here are not errors, they are the spread of the modes in $m$. Figure 2 $\mathrm{b}$ shows only the spread as the function of the clover coefficient. $m_{c}$, where the pion becomes massless, lies somewhere in the middle of the mode distribution, closer to its right (large mass) end. The average eigenmode locations and the 
upper end of the error bars in figure 2 a bracket $m_{c}$. Smearing the link removes most of the additive mass renormalization even for the Wilson action, reducing $m_{c} \sim-0.95$ for the thin link action to $m_{c} \sim-0.22$ for our case. Adding a clover term to the action further reduces the additive mass renormalization, and even larger clover terms induce a positive mass renormalization. At $\beta=5.8$ the additive mass renormalization is minimal for $c \approx 1.2$. This is also the value where the spread of the eigenmodes is minimal. To conclude, the results indicate that at $\beta=5.8$ (lattice spacing $a \simeq 0.15 \mathrm{fm}$ ) the low lying and doubler modes are well separated and explicit chiral symmetry breaking is minimized with clover coefficient $C=1.2$ with our fat link action.

The situation is less convincing at $\beta=5.7$ (lattice spacing $a \simeq 0.20 \mathrm{fm}$ ). Figure 3, again based on $408^{4}$ configurations, shows the distribution of the eigenmodes at $\beta=5.7$, clover coefficient $C=1.0,1.2$ and 1.4. Here the low lying modes are not as well separated than for $\beta=5.8$. For $C=1.0$ the distribution has a large tail extending towards negative quark masses. The situation is a bit better for $C=1.2$. Since the low lying modes are not well separated from the doublers, it makes no sense to calculate the spread of the distribution. The continuum description of chiral symmetry breaking is about to break down at $\beta=5.7$. If any of the distributions of figure 3 describes continuum physics, it is $C=1.2$ where the overall mass renormalization is close to zero and the low lying modes are best separated form the doublers.

Finally, at $\beta=5.55$ (lattice spacing $a \simeq 0.24 \mathrm{fm}$ ) Fig. 1 shows that the distribution of eigenmodes is broad and the low lying modes and doublers are completely mixed.

These figures show that it is not possible to make the lattice spacing greater than about $0.2 \mathrm{fm}$, and still retain the continuum-like description of chiral symmetry breaking using a clover-like action. Other, better tuned actions might perform better at large lattice spacing. Our exploratory studies with a hypercubic fixed point 13 action showed that the FP action is not better in terms of chiral symmetry breaking than the fat link clover action with $C=1.0$. Its dispersion relation was improved at lattice spacing $a=0.36 \mathrm{fm}$, and it (and the clover action) showed only a small amount of scale violation in hyperfine splittings at large lattice spacing. This just shows that scaling or near scaling of a few quantities does not guarantee that the physical mechanism responsible for chiral symmetry breaking is the same as in the continuum. This should serve as a warning sign for any calculations at large lattice spacing with actions of untested chiral properties.

In principle, one should optimize not the spread of the bare quark mass, but the spread of eigenmodes in terms of some physical observable, like the pion mass. However, at large fattening, the relation between the bare quark mass 
and the pion mass shows little variation with $C$.

The usual nonperturbative tuning of the clover term [20] attempts to optimize current algebra via PCAC relations. It is not clear to us how this is related to what we do, since it has been done so far only for thin link actions. We know that the optimal $C$ by our criterion for a thin link action is larger than the usual nonperturbative tuning, for example 2.5 vs 2.0 at $\beta=5.8$. Our criterion is based on a direct attack on low energy real eigenmodes. To the extent that the dynamics of QCD for light quark masses is dominated by this physics, we feel our optimization criterion is well founded.

We do not know if there is a connection between this tuning method and the Ginsparg-Wilson [21] realization of chiral symmetry on the lattice. The free field limit of these fat link actions is identical to the standard Wilson action, and so we are doing something different from improving the chiral properties of the interacting theory by improving the chiral properties of the free theory.

\section{Scaling Tests}

We have done a calculation of spectroscopy and matrix elements at lattice spacing $a T_{c}=1 / 4(\beta=5.7$ with the Wilson gauge action) using the fat link clover action with $C=1.2$. This is on the edge of the allowed range of lattice spacings according to our criterion of the last section. By itself, one lattice spacing is not a scaling test, but we can combine our results with those from other actions to compare the new action to them.

\subsection{Spectroscopy}

The spectroscopy measurement is entirely straightforward. The lattice volume was $8^{3} \times 24$.The data set was 80 lattices. We gauge fixed to Coulomb gauge and used a Gaussian independent particle source wave function $\psi(r)=$ $\exp \left(-\left(r / r_{0}\right)^{2}\right)$ with $r_{0}=2$. We used pointlike sinks projected onto low momentum states. We used naive currents $\left(\bar{\psi} \gamma_{5} \psi\right.$, etc.) for interpolating fields. The spectra appeared to be asymptotic (as shown by good (correlated) fits to a single exponential) beginning at $t \simeq 3-5$ and the best fits were selected using the HEMCGC criterion 22 .

Our fiducials for comparison are Wilson action and clover action quenched spectroscopy. We have tried to restrict the data we used for comparison to 

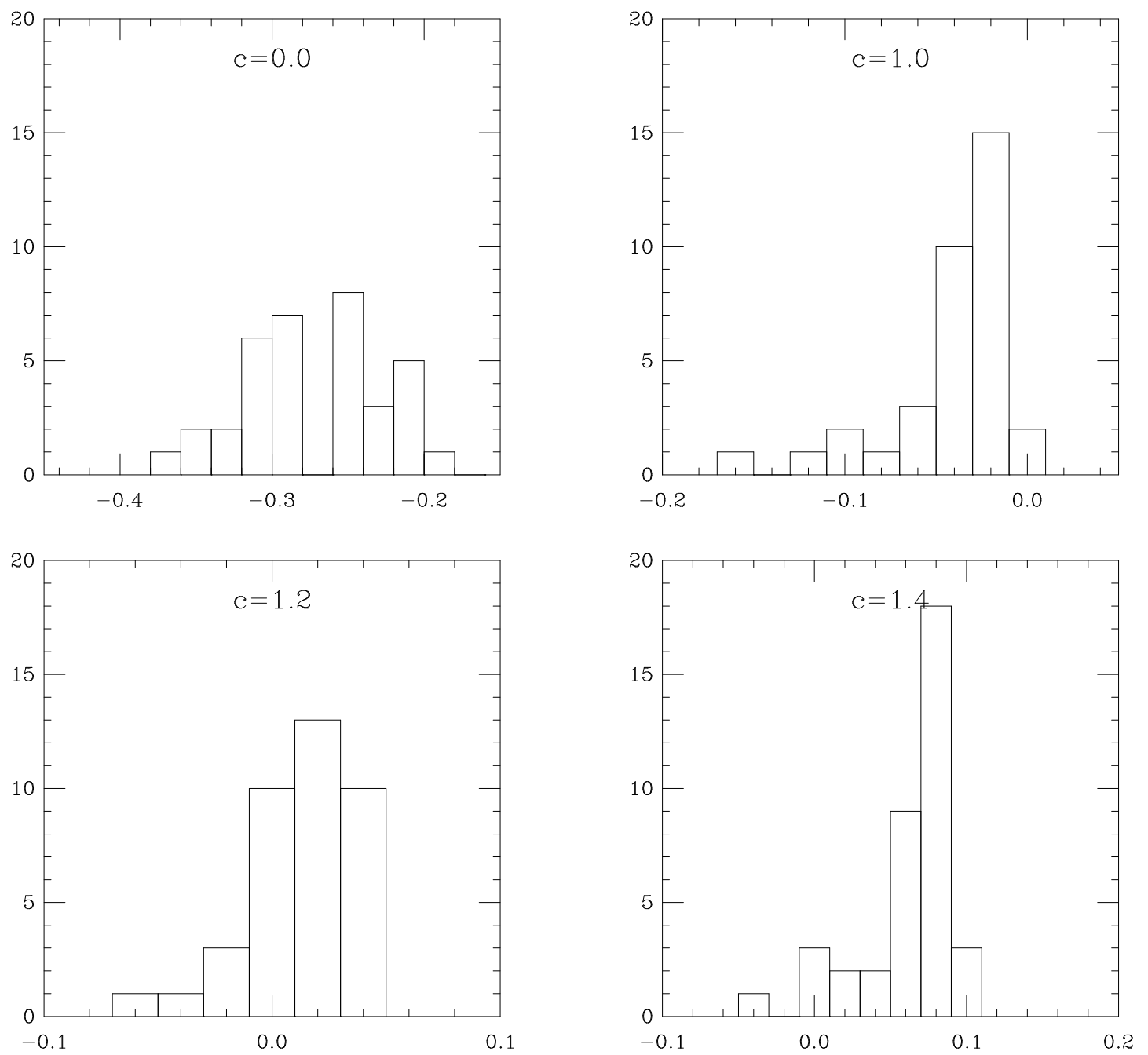

Figure 1: Locations of the real eigenmodes at $\beta=5.8$ of fat link Wilson action (a); fat link clover fermions $\mathrm{C}=1.0$ (b); $C=1.2$ (c); $C=1.4$ (d). 

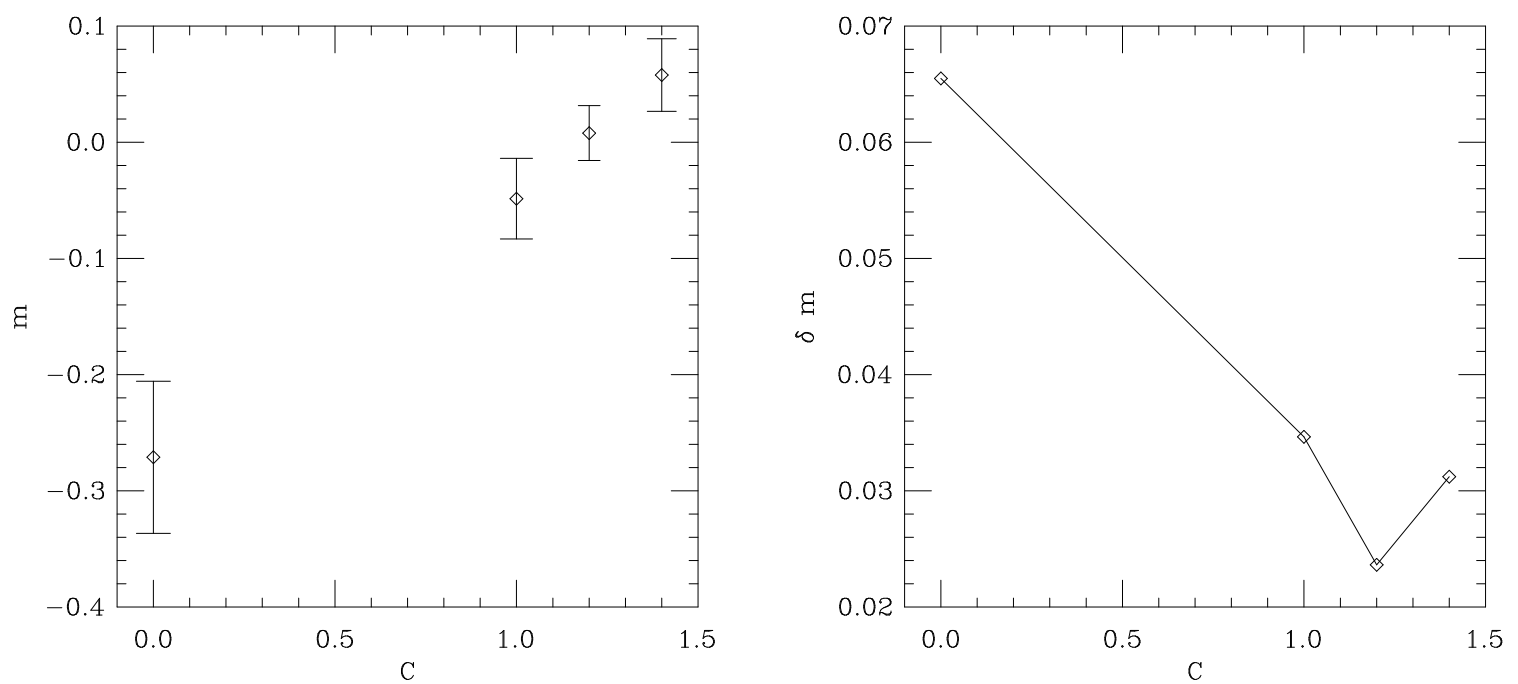

Figure 2: (a) The average real eigenmode location as the function of the clover coefficient at $\beta=5.8$. The error bars show the spread of the modes. (b) The spread of the modes from (a) as the function of the clover coefficient.

lattices with the proper physical volume.

We can roughly estimate the critical bare quark mass (at which the pion is massless) by linearly extrapolating $m_{\pi}^{2}$ to zero in $m_{0}$. We also estimated the critical bare mass using the PCAC relation

$$
\nabla_{\mu} \cdot\left\langle\bar{\psi} \gamma_{5} \psi(0) \bar{\psi} \gamma_{5} \gamma_{\mu} \psi(x)\right\rangle=2 m_{q}\left\langle\bar{\psi} \gamma_{5} \psi(0) \bar{\psi} \gamma_{5} \psi(x)\right\rangle .
$$

which, going to the lattice and following [23] is done by fitting the pseudoscalar source-pseudoscalar sink to

$$
P(t)=Z\left(\exp \left(-m_{\pi} t\right)+\exp \left(-m_{\pi}\left(N_{t}-t\right)\right)\right)
$$

and the pseudoscalar source-axial sink to

$$
A(t)=\frac{Z_{P}}{Z_{A}} \frac{2 m_{q}}{m_{\pi}} Z\left(\exp \left(-\left(m_{\pi} t\right)-\exp \left(-m_{\pi}\left(N_{t}-t\right)\right)\right)\right.
$$

to extract $m_{q}$. Fig. 5 shows the squared pion mass vs bare quark mass. The quark masses from the local pseudoscalar and axial currents are also shown.

Fits of the squared pion mass $\left(a m_{\pi}\right)^{2}=B\left(1 / \kappa-1 / \kappa_{c}\right)$ give $B=1.55(2)$ and $\kappa_{c}=0.12515(7)$. The quark mass is fit to $a m_{q}=A\left(1 / \kappa-1 / \kappa_{c}\right)$ and we find $A=0.447(7), \kappa_{c}=0.12515(6)$. In free field theory, we would expect 

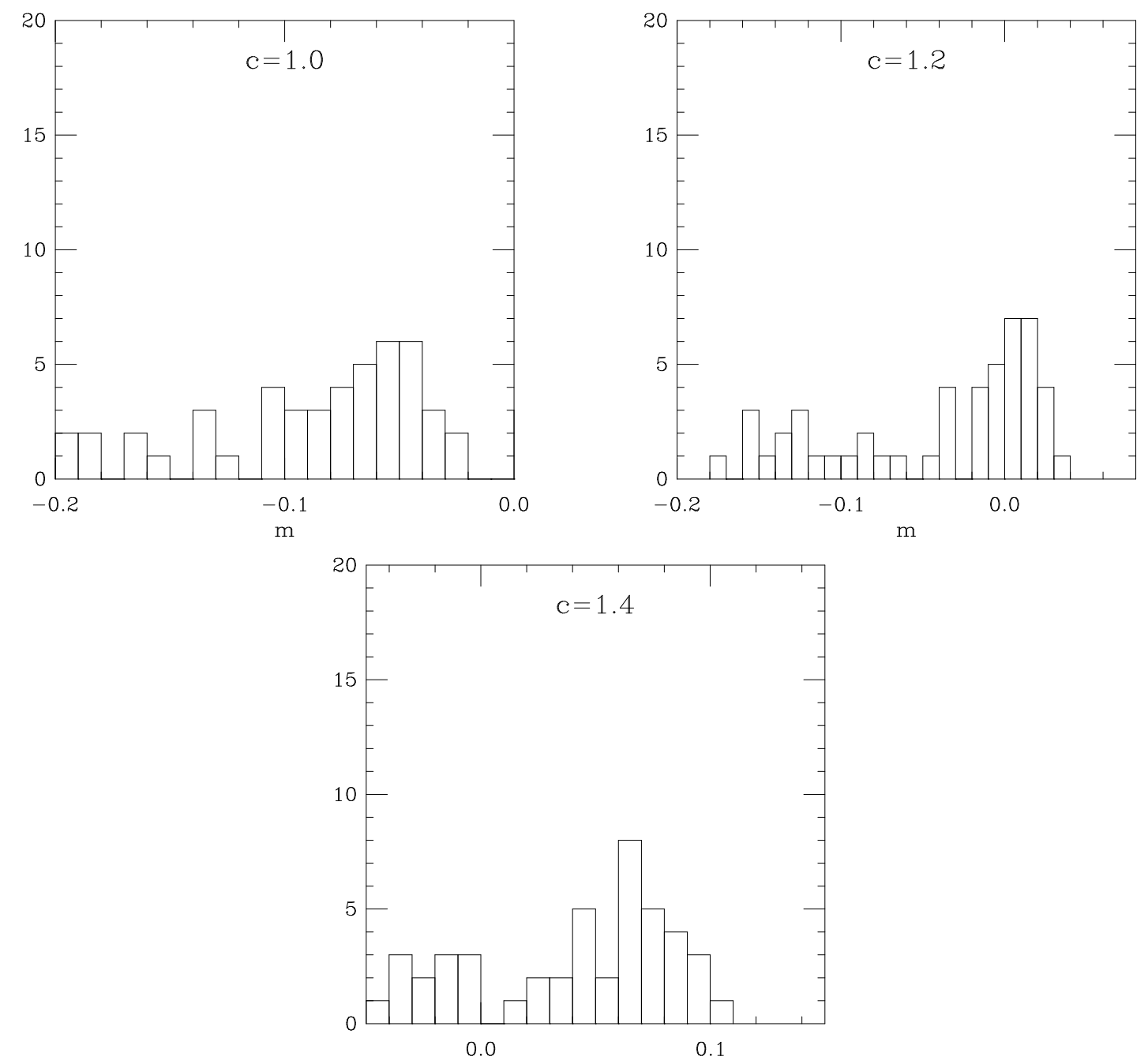

Figure 3: Locations of the real eigenmodes at $\beta=5.7$ of fat link clover fermions $\mathrm{C}=1.0(\mathrm{a}) ; C=1.2(\mathrm{~b}) ; C=1.4(\mathrm{c})$. 

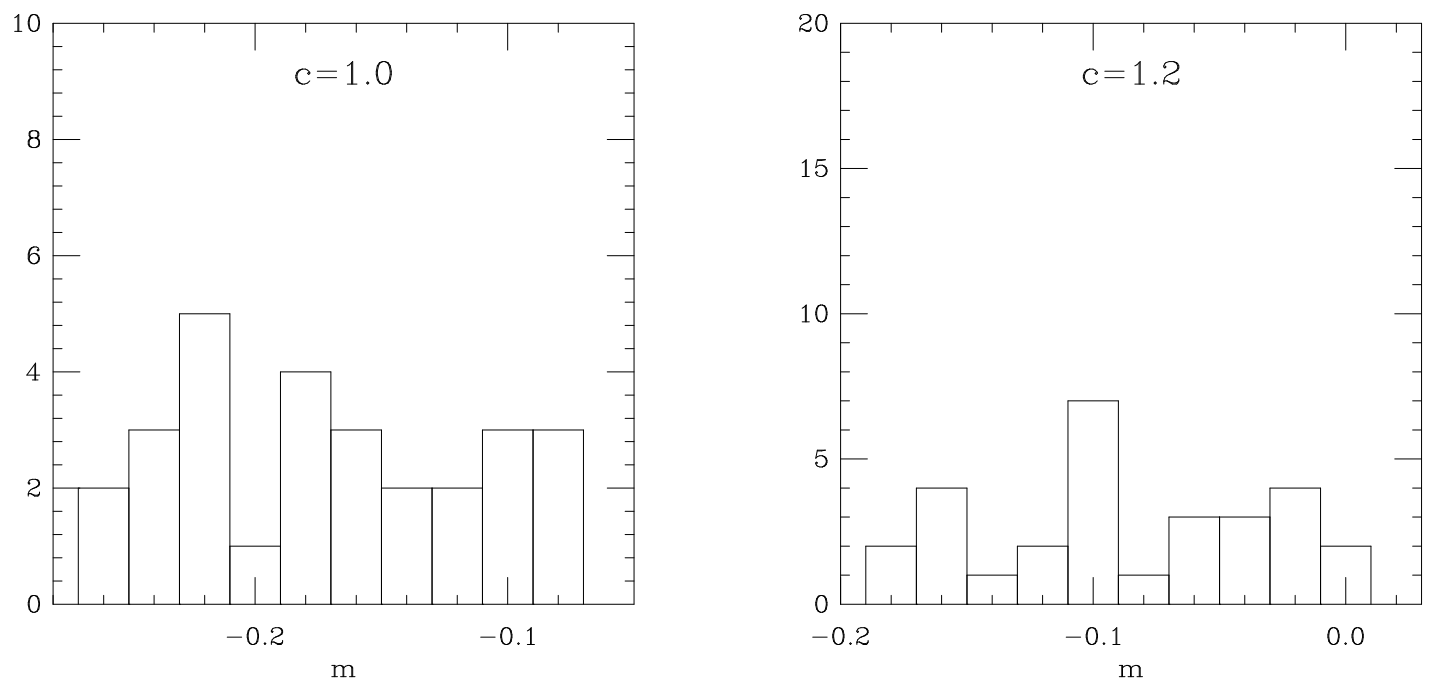

Figure 4: Locations of the real eigenmodes at $\beta=5.55$ of fat link clover fermions $\mathrm{C}=1.0(\mathrm{a}) ; C=1.2(\mathrm{~b})$.

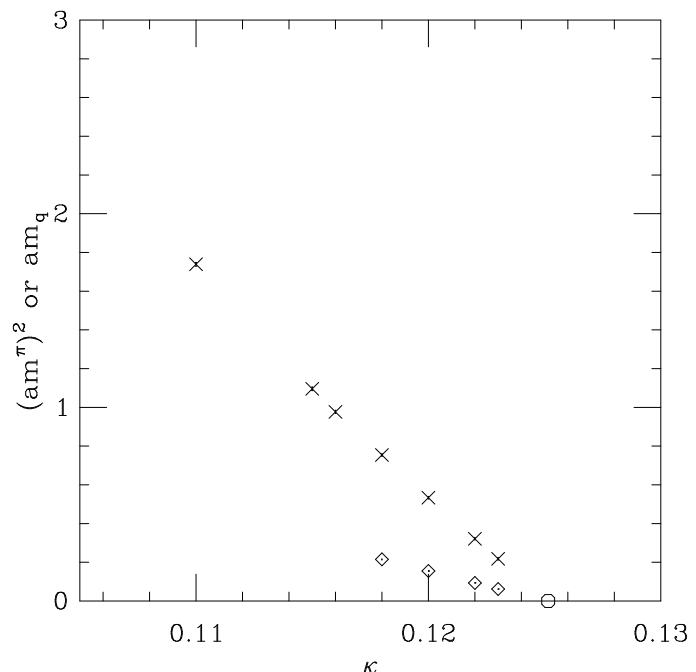

Figure 5: Bare squared pion mass (crosses) and quark mass from Eq. 12 (diamonds) vs hopping parameter for the $\mathrm{C}=1.2$ action. The octagon shows $\kappa_{c}$. 


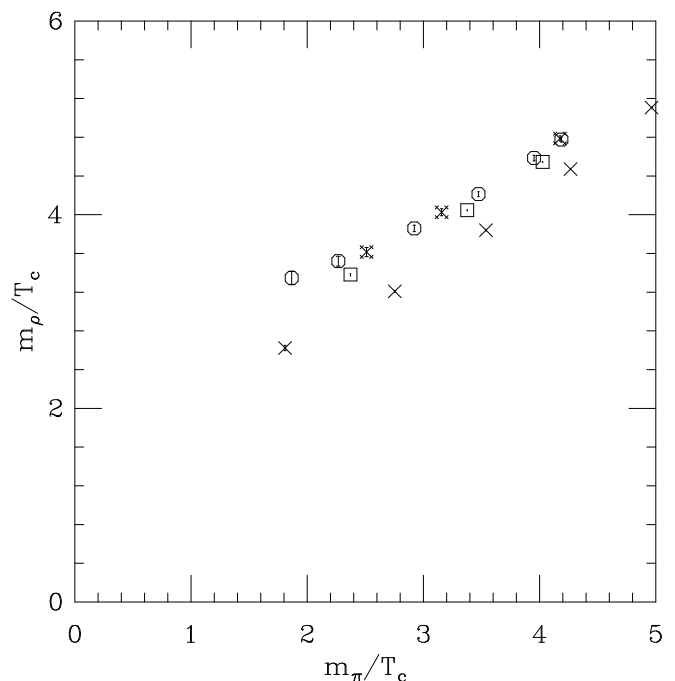

Figure 6: Octagons show $m_{\rho} / T_{c}$ vs. $m_{\pi} / T_{c}$ for the $\mathrm{C}=1.2$ action at $a T_{c}=1 / 4$. Also shown are Wilson action data, with crosses for $a T_{c}=1 / 4$, squares for $a T_{c}=1 / 8$, and fancy crosses for $a T_{c}=1 / 12$.

$A=1 / 2, \kappa_{c}=1 / 8$, and this is our first hint that perturbative corrections to bare quantities are small.

As a scaling test we compare $m_{\rho} / T_{c}$ and $m_{N} / T_{c}$ vs. $m_{\pi} / T_{c}$ for the $\mathrm{C}=1.2$ action in Figs. 6 and 7 .

We compare scaling violations in hyperfine splittings by interpolating our data to fixed $\pi / \rho$ mass ratios and plotting the $N / \rho$ mass ratio vs. $m_{\rho} a$. We do this at three $\pi / \rho$ mass ratios, $0.80,0.70$ and 0.60 , in Fig. 8 . In these figures the diamonds are Wilson action data in lattices of fixed physical size, $8^{3}$ at $\beta=5.7$ [24, $16^{3}$ at $\beta=6.0$ [25] $24^{3}$ at $\beta=6.3$ [26], and the crosses are data in various larger lattices: $16^{3}$ and $24^{3}$ at $\beta=5.7$ and $32^{3}$ at $\beta=6.17$ [24, $24^{3}$ at $\beta=6.0$ [25. When they are present the data points from larger lattices illustrate the danger of performing scaling tests with data from different volumes. The bursts are from the nonperturbatively improved clover action of Refs. [27] and [28]. The squares show the $\mathrm{C}=1.2$ action.

We give a table of masses from the $\mathrm{C}=1.2$ action in Table 1 .

We conclude that the fat link clover action has at least as good scaling behavior as the usual nonperturbatively tuned clover action. 


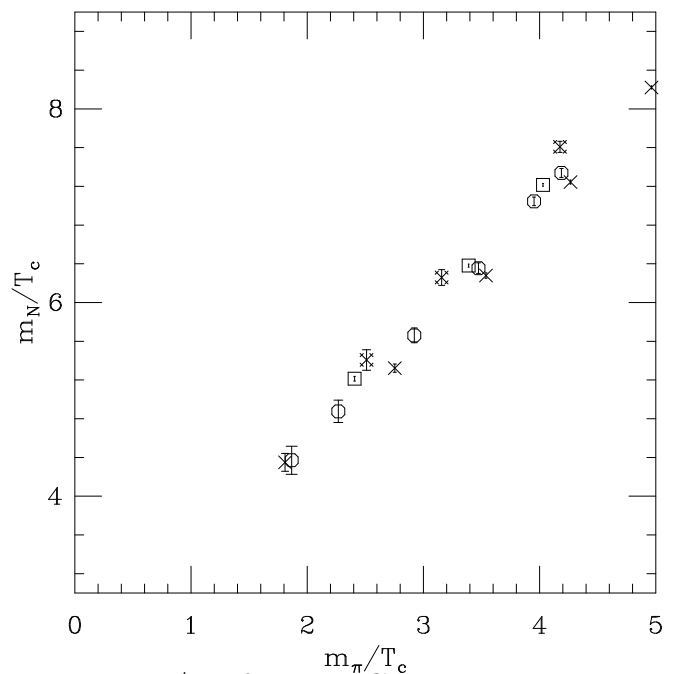

Figure 7: $m_{N} / T_{c}$ vs. $m_{\pi} / T_{c}$ for the $\stackrel{\mathrm{C}}{=}=1.2$ action and the Wilson action, labelled as in Fig. 6.

We also noticed that the new action seems to be more convergent than the thin link clover action: the same biconjugate gradient code needs about half as many steps to converge to the same residue as the usual thin link clover action, for the same $\pi / \rho$ mass ratio.

We did not encounter any exceptional configurations with the fat link action for $m_{\pi} / m_{\rho} \geq 0.56$.

\subsection{Dispersion Relations}

To view the dispersion relation, we first plot $E(p)$, the energy of the state produced with spatial momentum $\vec{p}$, as a function of $|\vec{p}|$. The result for the $\mathrm{C}=1.2$ action at $\kappa=0.118$ is compared to the free dispersion relation at $a T_{c}=$ $1 / 4$ in Fig. 9. It looks very similar to results from the Wilson action at the same lattice spacing-an entirely unsurprising result.

This behavior is quantified by measuring the squared speed of light, $c^{2}=$ $\left(E(p)^{2}-m^{2}\right) / p^{2}$, for $\vec{p}=(1,0,0)$. We do this by performing a correlated fit to the two propagators. The result is presented in Fig. 10 and shows the worsening of the dispersion relation at larger quark mass, characteristic Wilson/clover kinetic behavior. 

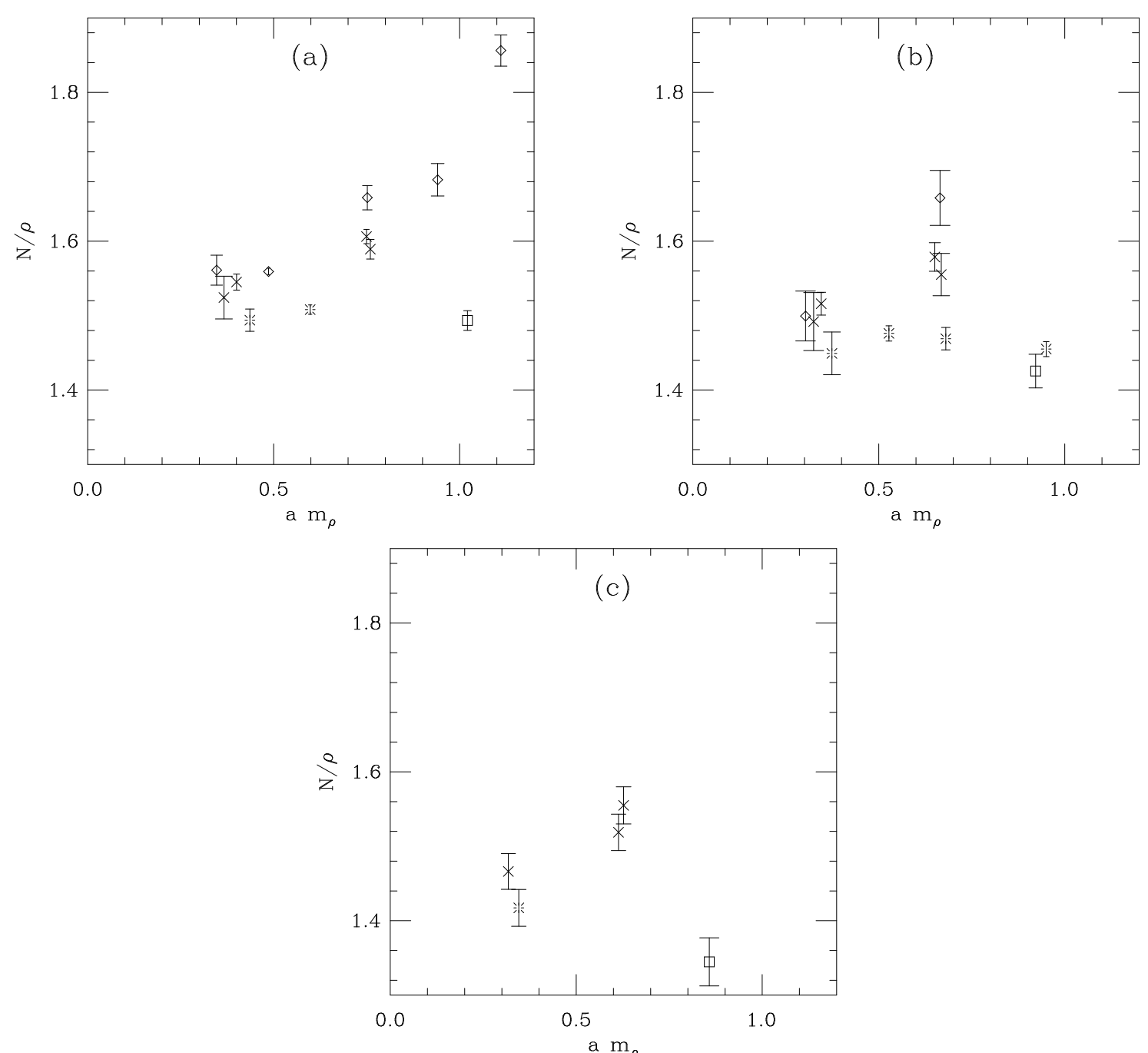

Figure 8: A scaling test for the $\mathrm{C}=1.2$ action (square) vs. Wilson actions on lattices of fixed physical size (diamonds) and larger volumes (crosses), and the nonperturbatively improved clover action (bursts). Data are interpolated to $\pi / \rho=0.80(\mathrm{a}), 0.70(\mathrm{~b})$, and $0.60(\mathrm{c})$. 


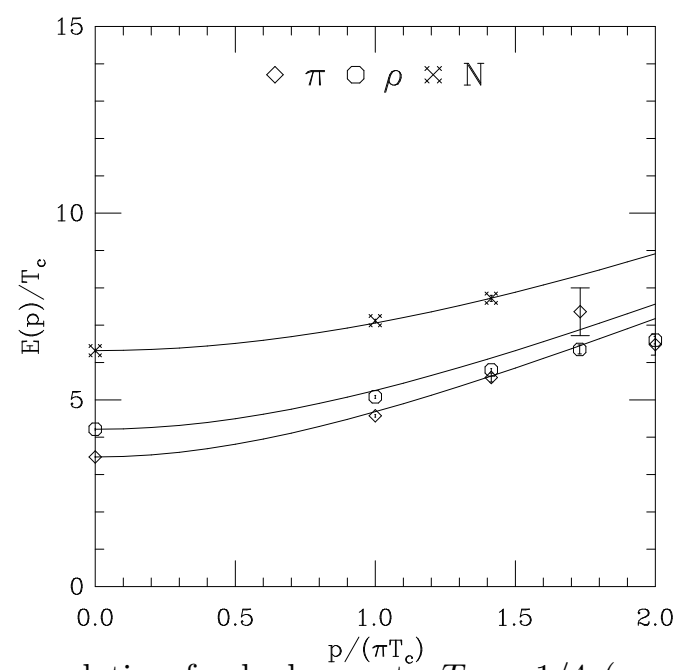

Figure 9: Dispersion relation for hadrons at $a T_{c}=1 / 4(a \simeq 0.18 \mathrm{fm})$ from the $\mathrm{C}=1.2$ action. The curves are the continuum dispersion relation for the appropriate (measured) hadron mass.

\begin{tabular}{|c|l|l|l|l|}
\hline$\kappa$ & PS & V & $\mathrm{N}$ & $\Delta$ \\
\hline 0.118 & $0.868(3)$ & $1.053(6)$ & $1.589(13)$ & $1.725(19)$ \\
0.120 & $0.730(4)$ & $0.965(8)$ & $1.415(19)$ & $1.593(22)$ \\
0.122 & $0.567(5)$ & $0.880(12)$ & $1.219(28)$ & $1.501(24)$ \\
0.123 & $0.467(6)$ & $0.837(17)$ & $1.093(36)$ & $1.432(27)$ \\
\hline
\end{tabular}

Table 1: Table of best-fit masses, $C=1.2, \beta=5.7$.

\subsection{Renormalization factors}

We now turn to a (rather naive) set of measurements of simple matrix elements.

Formally, the fat link action can be regarded as "just another" $O\left(a^{2}\right)$ improved action, if one assumes that the coefficient of the clover term is actually $C=1+O\left(g^{2}\right)$. In perturbation theory, which involves the vector potential $A_{\mu}$ rather than the link, the action shows the usual cancellation between the clover term and the scalar $\bar{\psi} \psi A$ term. The spectrum is $O\left(a^{2}\right)$; matrix elements using "rotated fields" $\psi \rightarrow(1-(\gamma \cdot D) / 2) \psi$ are $O\left(a^{2}\right)$ improved. Of course, the lattice-to-continuum renormalization Z-factors are different than the usual thin link clover Z-factors.

In principle, one could calculate all Z-factors using lattice perturbation theory. The Feynman rules differ from the rules for the usual clover action, only in that the vertices are multiplied by form factors, which are functions of the 

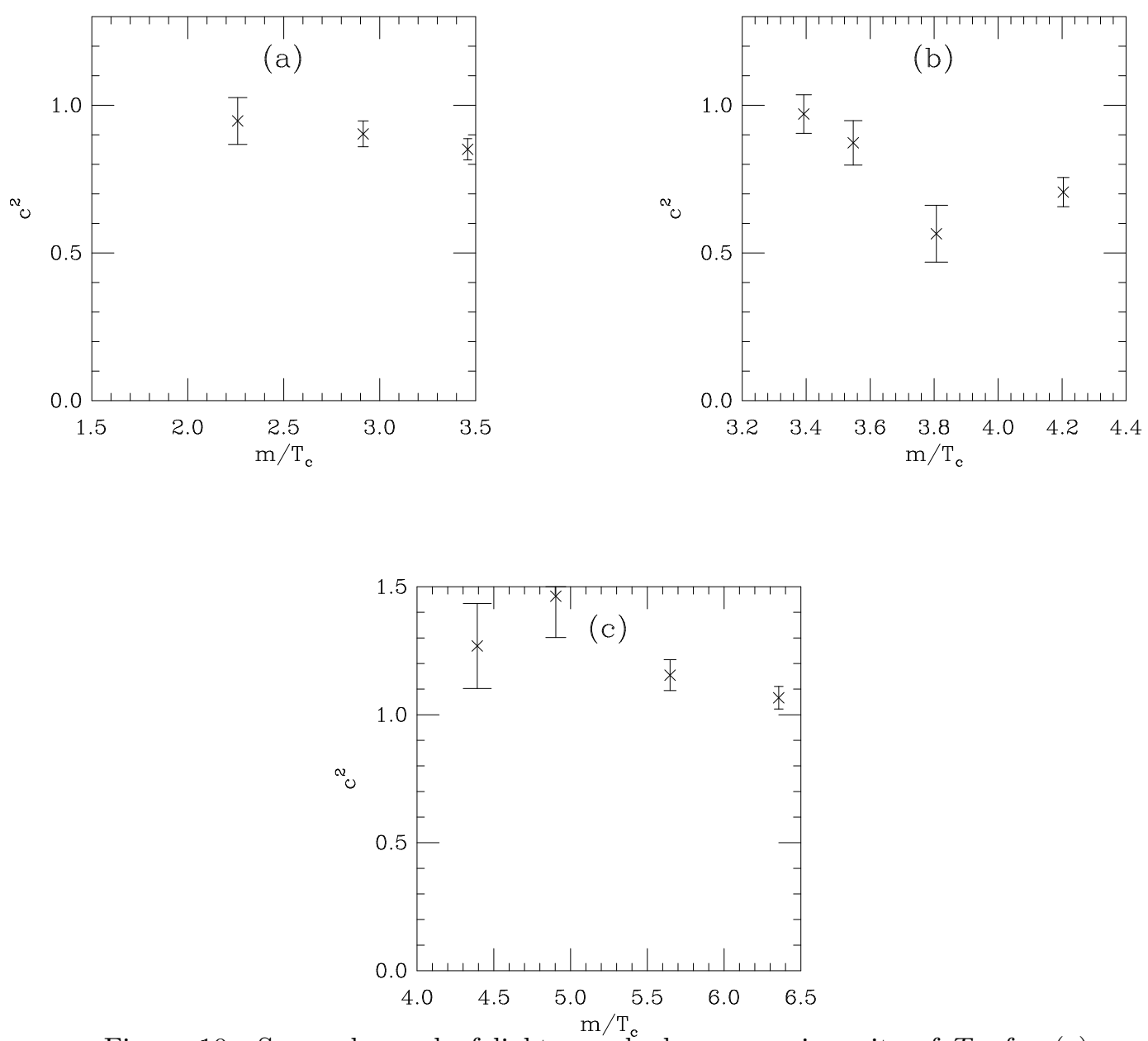

Figure 10: Squared speed of light vs. hadron mass in units of $T_{c}$, for (a) pseudoscalars, (b) vectors) and (c) protons, from the $\mathrm{C}=1.2$ action at $\beta=5.7$. 
gluonic momentum. We have not tried to do this yet. Since we are working at fairly strong coupling, we felt that it would be easier to compute the Z-factors nonperturbatively, beginning with the vector current and then computing the axial renormalization factor using Ward identities. Since this is just the first calculation using this action, we will restrict ourselves to the naive (local) currents.

We begin with the vector current. The conserved (Noether) current for the fat link action is the same as the Wilson current, just with fat links instead of thin links,

$$
J_{\mu}^{\text {cons }}(n)=\frac{1}{2}\left(\bar{\psi}(n)\left(\gamma_{\mu}-1\right) V_{\mu}(n) \psi(n+\hat{\mu})-\bar{\psi}(n+\hat{\mu})\left(\gamma_{\mu}+1\right) V_{\mu}^{\dagger}(n) \psi(n)\right) .
$$

This current is conserved but not improved. We choose to define the vector current Z-factor from the ratio of forward matrix elements

$$
Z_{V}=\frac{\langle\pi(T) \pi(0)\rangle}{\left\langle\pi(T) J_{0}(t) \pi(0)\right\rangle}
$$

for $0<t<T$. The local current is defined as $J_{\mu}^{l o c}(n)=\bar{\psi}(n) \gamma_{\mu} \psi(n)$. We measured the Z-factors using 20 configurations on a periodic $8^{3} \times 24$ lattice with $T=10$ and averaged $9>t>1$. The results of a jacknife analysis are shown in Table 2 .

The fact that $Z^{\text {cons }} \neq 1$ is a finite size effect. In the numerator of Eqn. 14 both quarks can propagate in both time directions around the torus, but the forwards and backwards paths contribute differently to the denominator. We correct this phenomenologically by computing the ratio $Z^{l o c} / Z^{c o n s}$ and display it in the table.

Lattice perturbation theory which correctly takes into account the residue of the pole of the massive quark 29] predicts that this ratio is equal to

$$
Z_{V}=\frac{1-6 \kappa}{2 \kappa} \hat{Z}_{V}
$$

where the $(1-6 \kappa)$ factor is the residue (recall $\left.\kappa_{c}=.125\right)$ and $\hat{Z}_{V}=1+a g^{2}+\ldots$. As Table 2 shows, the correction to the tree level formula, $\hat{Z}$, which is what is usually quoted as the Z-factor, differs from unity by less than than two per cent at $\kappa=0.123$.

We also attempted to measure the axial vector current renormalization using the chiral Ward identity [30]. With 50 lattices, we only had a signal at $\kappa=0.118$, where we found $Z_{A}=1.210(8)$. This is again quite close to the pure kinetic result of 1.237. Dividing out the kinematic factors, this would give $\hat{Z}_{A}=0.978$. 


\begin{tabular}{|c|l|l|l|l|}
\hline$\kappa$ & $Z^{\text {cons }}$ & $Z^{\text {loc }}$ & $Z^{\text {loc }} / Z^{\text {con }}$ & $\frac{1-6 \kappa}{2 \kappa}$ \\
\hline 0.118 & $1.020(1)$ & $1.253(2)$ & 1.228 & 1.237 \\
0.120 & $1.032(2)$ & $1.185(4)$ & 1.148 & 1.166 \\
0.122 & $1.054(5)$ & $1.129(6)$ & 1.071 & 1.098 \\
0.123 & $1.071(6)$ & $1.127(8)$ & 1.052 & 1.065 \\
\hline
\end{tabular}

Table 2: Vector current renormalization factors, $C=1.2, \beta=5.7$.

At $\beta=6.0$, Ref. [31] found $\hat{Z}_{V}=0.824, \hat{Z}_{A}=1.09$ for the usual clover action, and improved operators.

It would be very interesting to measure the mixing of left- and right-handed operators (for $B_{K}$ ) in this action.

\section{Conclusions}

We have shown that via a combination of fattening the links and tuning the magnitude of the clover term, it is possible to optimize the chiral behavior of the clover lattice fermion action. The example we presented reduces the spread in the low energy real eigenmodes by about a factor of three in units of the squared pion mass, compared to the usual Wilson action. We fixed the fattening and varied the clover coefficient, but it is clear that a real optimization would involve varying both factors.

The same procedure can be applied to the usual clover action, without fat links, but the value of the clover term $C$ which minimizes the spread of real eigenmodes due to instantons is so large that new kinds of configurations, whose singular behavior is unrelated to fermion modes sitting on individual instantons, become exceptional [5]. Thus this choice of action does not represent a good choice for improvement.

The method of construction exposes an apparent fundamental upper limit for the lattice spacing in a QCD simulation of about $0.2 \mathrm{fm}$. At larger lattice spacing, the doubler modes and the low energy modes do not show a clean separation, and the mechanism of chiral symmetry breaking is qualitatively different than in the continuum. The Wilson and clover actions, and the one improved action we tested, the hypercubic action of Ref. [13], failed at lattice spacing $0.24 \mathrm{fm}$. This test does not apply to actions involving heavy quarks, for which chiral symmetry is (presumably) not important, but before any other improved action can be said to reproduce continuum light hadron physics at some lattice spacing, it should show a separation between the spectrum of its 
near low energy modes and its doubler modes.

The "fat clover" action we have presented in this paper, as an example of an action with improved chiral properties, has many other nice properties as well. It appears to exhibit scaling of hyperfine splittings at $0.2 \mathrm{fm}$. The action is quite insensitive to the UV behavior of the underlying gauge field. It has Z-factors for simple matrix elements which are very close to unity. And finally, the amount of resources required to construct propagators is halved compared to the usual clover action, at equivalent parameter values. It would be trivial to modify any existing clover code to improve it in the way we have described. Of course, its kinetic properties, including the dispersion relation, artifacts at large $a m_{q}$, and power law scaling violations in matrix elements, are unchanged from the standard clover action's.

\section{Acknowledgements}

We would like to thank the Colorado high energy experimental groups for allowing us to use their work stations. Part of the computing was done on the Origin 2000 at the University of California, Santa Barbara. This work was supported by the U.S. Department of Energy.

\section{References}

[1] Cf. the review talk of P. van Baal at Lattice 97, Nucl. Phys, B (Proc. Suppl.) 63 (1998) 126.

[2] Cf. D. Diakanov, Lectures at the Enrico Fermi School in Physics, Varenna, 1995, hep-ph/9602375; T. Schäfer and E. V. Shuryak, Rev. Mod. Phys. 70 (1998) 323.

[3] A. Hasenfratz and C. Nieter, hep-lat/9806026.

[4] W. Bardeen, A. Duncan, E. Eichten, G. Hockney and H. Thacker, Phys. Rev. D57 (1998) 1633; W. Bardeen, A. Duncan, E. Eichten and H. Thacker, Phys. Rev. D57 (1998) 3890; hep-lat/9606002.

[5] T. DeGrand, A. Hasenfratz, and T. Kovacs, in preparation.

[6] M. Falcioni, M. Paciello, G. Parisi, B. Taglienti, Nucl. Phys. B251[FS13], 624 (1985). M. Albanese, et. al. Phys. Lett. B192, 163 (1987). 
[7] M.-C. Chu, J. M. Grandy, S. Huang and J. W. Negele, Phys. Rev. D49 (1994) 6039.

[8] T. Blum, et al., Phys. Rev. D55, 1133 (1997).

[9] K. Orginos and D. Toussaint, hep-lat/9801020.

[10] J.-F. Lagaë and D. K. Sinclair, Nucl. Phys. B(Proc. Suppl.) 63 (1998) 892.; hep-lat/9806014.

[11] T. DeGrand, A. Hasenfratz, T. Kovács, Phys. Lett. B420 (1998) 97.

[12] W. Bietenholz and U. J. Wiese, Nucl. Phys. B464 (1996) 319; T. DeGrand, A. Hasenfratz, P. Hasenfratz, P. Kunszt, F. Niedermayer, Nucl. Phys. B (Proc. Suppl.) 53, 1997, 942; W. Bietenholz, et al., Nucl. Phys. B(Proc. Suppl.) 53 (1997) 921; K. Originos, et al., contribution to Lattice '97, heplat/9709100, Nucl. Phys. B (Proc. Suppl.) 63 (1998) 904; C. B. Lang and T. K. Pany, hep-lat/9707024, Nucl. Phys. B513 (1998) 645.

[13] T. DeGrand, COLO-HEP-397, hep-lat/9802012.

[14] P. Hasenfratz, V. Laliena and F. Niedermayer, hep-lat/9801021; P. Hasenfratz, hep-lat/9702007.

[15] J. Smit and J. Vink, Nucl. Phys. B286 (1987) 485, J. Vink, Nucl. Phys. B307 (1988) 549.

[16] H. Simma, D. Smith, Low-lying eigenvalues of the improved Wilson-Dirac operator in QCD, hep-lat/9801025.

[17] R. G. Edwards, U. M. Heller, R. Narayanan, Nucl. Phys. B522 (1998) 285.

[18] H. Kluberg-Stern, A. Morel, and B. Petersson, Phys. Lett. B114 (1982) 152.

[19] R. G. Edwards, U. M. Heller, R. Narayanan, Spectral flow, chiral condensate and topology in the lattice, hep-lat/9802016.

[20] M. Lüscher, S. Sint, R. Sommer, and P. Weisz, Nucl. Phys. B502 (1996) 365

[21] P. Ginsparg and K. Wilson, Phys. Rev. D25 (1982) 2649.

[22] K. M. Bitar, et al., Phys. Rev. D42 (1990) 3794.

[23] C. Bernard, et al., Phys. Rev. D45 (1992) 3854.

[24] F. Butler, H. Chen, J. Sexton, A. Vaccarino, and D. Weingarten, Nucl. Phys. B430 (1994) 179. 
[25] M. Göckeler, et al. Phys. Lett. B391 (1997) 388.

[26] M. Guagnelli, et al., Nucl. Phys. B378 (1992) 616.

[27] M. Göckeler, et. al., hep-lat/9707021, Phys. Rev. D57 (1998) 5562.

[28] R. G. Edwards, U. M. Heller and T. R. Klassen, hep-lat/9711052.

[29] C. Bernard, J. Labrenz, and A. Soni, Phys. Rev. D49 (1994) 2536; G. P. Lepage and P. Mackenzie, Phys. Rev. D48 (1993) 2250.

[30] M. Bochicchio, et. al., Nucl. Phys. B262 (1985) 331; L. Karsten and J. Smit, Nucl. Phys. B183 (1981) 103. L. Maiani and G. Martinelli, Phys. Lett. B178 (1986) 285.

[31] G. Martinelli, S. Petrarca, C. T. Sachrajda, Phys. Lett. B311 (1993) 241. 